\title{
Collected Transatlantic Experience From the PERICLES Registry: Use of Chimney Grafts to Treat Post-EVAR Type la Endoleaks Shows Good Midterm Results
}

(C) The Author(s) 2018

Reprints and permissions:

sagepub.com/journalsPermissions.nav

DOI: $|0.1| 77 / 15266028 \mid 878294$

www.jevt.org

\author{
Sonia Ronchey, MD, PhD', Stefano Fazzini, MD, PhD'iD, Salvatore Scali, MD², \\ Giovanni Torsello, MD ${ }^{3}$, Paul Kubilis, MD ${ }^{2}$, Frank Veith, MD ${ }^{4,5}$, \\ Konstantinos P. Donas, $\mathrm{MD}^{3}$, Felice Pecoraro, $\mathrm{MD}^{4,6}$, and Nicola Mangialardi, $\mathrm{MD}^{7}$
}

\begin{abstract}
Purpose: The aim of this retrospective analysis was to evaluate the performance of the chimney (ch) technique in the treatment of type la endoleaks after standard endovascular aneurysm repair (EVAR). Methods: Between January 2008 and December 2014, 517 chEVAR procedures were performed in 13 US and European vascular centers (PERICLES registry). Thirty-nine patients (mean age 76.9 $\mathbf{7}$.I years; 33 men) were treated for persistent type la endoleak and had computed tomography angiography or magnetic resonance angiography follow-up at $>1$ month. Endurant abdominal stent-grafts were used in the 20 cases. Single chimney graft placement was performed in 18 (46\%) patients and multiple in 2I (54\%). Overall, 70 visceral vessels were targeted for revascularization. Results: Technical success was achieved in 35 (89.7\%) cases; 3 persistent type la endoleaks and I chimney graft occlusion were detected within the first 30 days. Thirty-day mortality was $2.6 \%$. Two other deaths (not aneurysm related) occurred during a mean follow-up of 21.9 months (0.23-71.3). Primary patency of the chimney grafts was $94.3 \%$ at 36 months. In a subgroup analysis comparing Endurant to other stent-grafts, no significant differences were observed regarding persistent endoleak [I/20 (5\%) vs $2 / 19(\mathrm{I} / \%), \mathrm{p}=0.6$ ] or reintervention [I/20 (5\%) vs 0/I9 (0\%)]. Conclusion: The present series demonstrates that chEVAR in the treatment of post-EVAR type la endoleaks has satisfactory results independent of the abdominal and chimney graft combinations. Midterm results show that chEVAR is an effective method for treating type la endoleaks.
\end{abstract}

\section{Keywords}

abdominal aortic aneurysm, chimney graft, chimney technique, endoleak, endovascular aneurysm repair, juxtarenal aortic aneurysm, parallel graft, pararenal aortic aneurysm, periscope graft, snorkel graft

\section{Introduction}

Type Ia endoleak after previous endovascular aneurysm repair (EVAR) has an incidence of $3.5 \%$ to $15.5 \%$ and is due to inadequate proximal sealing of the graft. ${ }^{1,2}$ It occurs more commonly when the graft is implanted outside the instructions for use or in complex anatomy (short, conical, large diameter, or severely angulated neck). However, even when the first implantation is performed in favorable anatomy, over time proximal neck degeneration can lead to sac reperfusion owing to lack of sealing and/or graft migration. ${ }^{3-6}$ Treatment of type Ia endoleak is mandatory as sac reperfusion is responsible for increasing aortic diameter and the risk of rupture.
'Department of Vascular Surgery, San Filippo Neri Hospital, Rome, Italy ${ }^{2}$ Division of Vascular Surgery and Endovascular Therapy, University of Florida, Gainesville, FL, USA

${ }^{3}$ Department of Vascular Surgery, St Franziskus Hospital Münster, Germany ${ }^{4}$ Cardiovascular Surgery Unit, University Hospital Zurich, Switzerland ${ }^{5}$ New York University Medical Center, New York, NY, USA

'University of Palermo, Vascular Surgery Unit, AOUP "P. Giaccone", Palermo, Italy

${ }^{7}$ Department of Vascular Surgery, "San Camillo-Forlanini" Hospital, Rome, Italy

Corresponding Author:

Stefano Fazzini, Department of Vascular Surgery, San Filippo Neri

Hospital, Via Martinotti 20, Rome, 00I35, Italy.

Email: dr.stefano.fazzini@gmail.com 
Table I. Patient Demographics, Comorbidities, Indications, and Preoperative Anatomic Characteristics. ${ }^{a}$

\begin{tabular}{lr}
\hline Age, y & $76.9 \pm 7.1$ \\
Men & $33(85)$ \\
ASA III & $20(5 \mathrm{I})$ \\
ASA IV & $19(49)$ \\
Comorbidities & \\
Diabetes & $10(26)$ \\
Hypertension & $37(95)$ \\
Dyslipidemia & $21(54)$ \\
Coronary artery disease & $21(54)$ \\
Chronic lung disease & $20(5 \mathrm{I})$ \\
Peripheral artery disease & $6(2 \mathrm{I})$ \\
Congestive heart failure & $13(33)$ \\
Chronic renal insufficiency & $13(33)$ \\
Aneurysm features & \\
Juxtarenal & $32(82)$ \\
Pararenal & $7(18)$ \\
Infrarenal neck diameter, mm & $27.0 \pm 5.2$ \\
Infrarenal neck length, mm & $3.9 \pm 4.0$ \\
Diameter, mm & $71.5 \pm 29.0$ \\
\hline
\end{tabular}

Abbreviations: AAA, abdominal aortic aneurysm; ASA, American Society of Anesthesiologists.

${ }^{a}$ Continuous data are presented as the means \pm standard deviation; categorical data are given as the counts (percentage).

Open conversion with graft explantation is a highly risky procedure, especially in fragile patients, and has a mortality $>10 \%$ even in experienced centers. ${ }^{7-9}$ Endovascular options include the use of cuffs, Palmaz stents, endoanchors, coils, and glues; these approaches have a perioperative mortality close to zero but unsatisfactory late results in terms of reintervention and risk of rupture. ${ }^{10-12}$

When it is necessary to create a new neck above the renal arteries, custom fenestrated devices and chimneys are the only options. The choice is strictly related to the anatomy, clinical setting (ie, urgent/emergency), centerspecific experience, material availability, and risk profile of the patient.

The aim of the present study was to analyze the outcomes of type Ia endoleak treatment using chimney EVAR (chEVAR) as collected in the PERICLES Registry (PERformance of the chimney technique for the treatment of Complex aortic pathoLogiES). ${ }^{13}$

\section{Methods}

\section{Study Cohort}

Between January 2008 and December 2014, 517 chEVAR procedures were performed in 13 US and European vascular centers, which all had experience treating $>10$ patients with complex abdominal aortic pathologies using this technique. Of these, 39 patients (mean age $76.9 \pm 7.1$ years; 33 men) were treated in elective or urgent procedures for type Ia endoleak and had at least 1 postoperative computed tomography angiography (CTA) or magnetic resonance angiography (MRA) scan beyond 1 month post EVAR. The original aortic aneurysms were juxtarenal in 32 (82\%) and pararenal in $7(18 \%)$. The endografts used in the initial EVAR procedure had suprarenal fixation in $29(74 \%)$ and infrarenal fixation in $10(26 \%)$.

The patients provided informed consent for the chEVAR procedure. Mean time from the previous EVAR to chEVAR was $7.0 \pm 1.3$ years. All the type Ia endoleaks were related to neck degeneration with migration $\leq 5 \mathrm{~mm}$ in $14(36 \%)$ cases or $>5 \mathrm{~mm}$ in $25(64 \%)$. All patients were considered highrisk candidates for open conversion (ie, banding or endograft explantation with aortic repair) and were classified as American Society of Anesthesiologists class III/IV because of severe comorbidities. Thirty (77\%) patients were asymptomatic and treated in the elective setting, while $9(23 \%)$ were treated urgently owing to abdominal/lumbar pain. The $\mathrm{CT}$ scans in these latter patients showed rapid increase in diameters and signs of impending rupture (blister, thin wall). Patient characteristics and preoperative anatomic characteristics are summarized in Table 1.

\section{Treatment Procedure}

The selection of chimney graft (CG) type [self-expanding covered stents (SECS) or balloon-expandable covered stents (BECS) with bare metal stents (BMS)] and aortic stent-graft was based on each institution's clinical practice and the surgeon's preference, as were CG configuration (chimney/ snorkel or periscopes), access sites, and main graft oversizing (range $25 \%$ to $35 \%$ ). All commercially available abdominal and even thoracic stent-grafts (Table 2) were used in different combinations with CGs. The most often implanted aortic cuff $(20,51 \%)$ was the Endurant (Medtronic, Minneapolis, MN, USA). Single CGs were placed in 18 (46\%) patients and multiple in 21 (54\%), mostly doubles $(14 / 21)$, for a total of 70 target arteries revascularized. Most of the CGs were placed in the renal arteries (57/70, 81\%), including 1 accessory renal artery.

\section{Antiplatelet Therapy and Follow-up Protocols}

Follow-up strategies and medication protocols were similar for all centers and predefined by the PERICLES Registry collaborators. The recommended postoperative antiplatelet therapy was aspirin $(100 \mathrm{mg})$ and clopidogrel $75 \mathrm{mg}$ daily for at least 2 months; clopidogrel $75 \mathrm{mg}$ alone was used in patients under anticoagulation. All patients had a CTA during the month after operation and were scheduled for outpatient follow-up with physical examination and CTA at 6 and 12 months and annually thereafter. Another CTA was performed at 3 months if type Ia endoleak 
Table 2. Stent-Grafts and Intraoperative Data.

\begin{tabular}{lc}
\hline Device & \\
Endurant & $20(5 \mathrm{I})$ \\
Zenith & $7(18)$ \\
C-TAG & $4(10)$ \\
Excluder & $3(8)$ \\
E-Vita abdominal & $2(5)$ \\
Zenith TX2 & $2(5)$ \\
Other & $1(3)$ \\
Chimney grafts (n=70) & \\
Right renal artery & $25(36)$ \\
Left renal artery & $31(44)$ \\
Superior mesenteric artery & $9(13)$ \\
Celiac trunk & $4(6)$ \\
Accessory renal artery & $1(1)$ \\
Types of chimney grafts & \\
Self-expanding covered stent & $18(46)$ \\
Balloon-expandable covered stent & $18(46)$ \\
Balloon-expandable bare metal stent & $3(8)$ \\
Endolining bare metal stent & $10(26)$ \\
Chimneys per patient & \\
I & $18(46)$ \\
2 & $14(36)$ \\
3 & $4(10)$ \\
4 & $3(8)$ \\
Intraoperative data & $74.8 \pm 8.2$ \\
Procedure time, min & $7(18)$ \\
Fluoroscopy time, min & $4(10)$ \\
Contrast volume, mL & $3(8)$ \\
Type I endoleak & $6(15)$ \\
$\quad$ la & $231.2 \pm 84.1$ \\
Treated type la/lb endoleak & \\
\hline & \\
\hline &
\end{tabular}

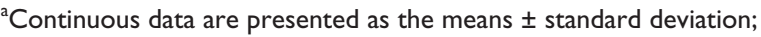
categorical data are given as the counts (percentage).

was seen at completion angiography. Contrast-enhanced ultrasound associated with a native CT or, alternatively, MRA was used in case of renal insufficiency.

\section{Outcomes}

The primary outcome was technical success, defined as successful main and parallel graft deployment at the intended site, patency of the CGs, and absence of type Ia endoleak at completion angiography. The secondary outcomes were 30-day mortality, the incidence of type Ia endoleak, primary patency of the CGs, and freedom from reintervention.

\section{Statistical Analysis}

Continuous data are presented as the means \pm standard deviation; categorical data are given as the counts (percentage). Kaplan-Meier analysis was used to estimate the survival rate
Table 3. Outcomes. ${ }^{a}$

\begin{tabular}{|c|c|}
\hline \multicolumn{2}{|l|}{ Follow-up, mo } \\
\hline Time to last CTA/MRA & $21.9(0.23-72.3)$ \\
\hline Time to death or censoring & $7.3(0.03-50.0)$ \\
\hline Mortality & 3 \\
\hline 30-day & 1 \\
\hline \multicolumn{2}{|l|}{ Cause of death } \\
\hline Cardiac & I \\
\hline Pneumonia & 2 \\
\hline Any complication & 8 \\
\hline Late type I endoleak & 3 \\
\hline Treated late type I endoleak (Endurant) & 1 \\
\hline Chimney occlusion & 4 \\
\hline \multicolumn{2}{|l|}{ Postoperative AAA anatomic features } \\
\hline Infrarenal neck length, $\mathrm{mm}$ & $20.4 \pm 4.2$ \\
\hline Maximum AAA diameter, $\mathrm{mm}$ & $69.9 \pm 27.5$ \\
\hline Change in AAA diameter, $\mathrm{mm}$ & -1.6 \\
\hline
\end{tabular}

and CG patency; the estimated rates are presented with the 95\% confidence intervals (CI). The threshold of statistical significance was $\mathrm{p}<0.05$. The statistical analysis was performed with SPSS software (version 22.0; IBM Corporation, Armonk, NY, USA).

\section{Results}

Technical success was achieved in 35 (90\%) cases. No evidence of any type of endoleak was observed in the postoperative control (Table 2), but $3(8 \%)$ persistent type Ia endoleaks and 1 (3\%) CG occlusion were detected within the first 30 days (Table 3). Two of the endoleaks (gutters-related with a stable diameter) were treated conservatively under radiologic surveillance, while 1 patient with triple $\mathrm{CG}$ placement and increasing aortic diameter was successfully treated using Onyx embolization 7 months postoperatively (freedom from reintervention $97.4 \%$ ).

No early/late procedure-related death occurred. However, 1 patient died due to acute cardiac failure within the first month (30-day mortality $2.6 \%$ ), while 2 patients died due to pneumonia during follow-up (overall mortality $7.7 \%$; Figure 2A.

The mean follow-up of this patient cohort was 21.9 months (range $0.23-72.3$ ). Primary patency of the CGs was $94.3 \%(66 / 70)$ at 36 months. Based on the type of CG, the estimated overall primary patency of CGs at 24 months was 86.1\% (Figure 2B): $100 \%$ for SECS and $77.2 \%$ for BECS and BMS. No difference was observed in CG primary patency considering the different types of main graft (Endurant 85\% vs 87.5\% for other models). 

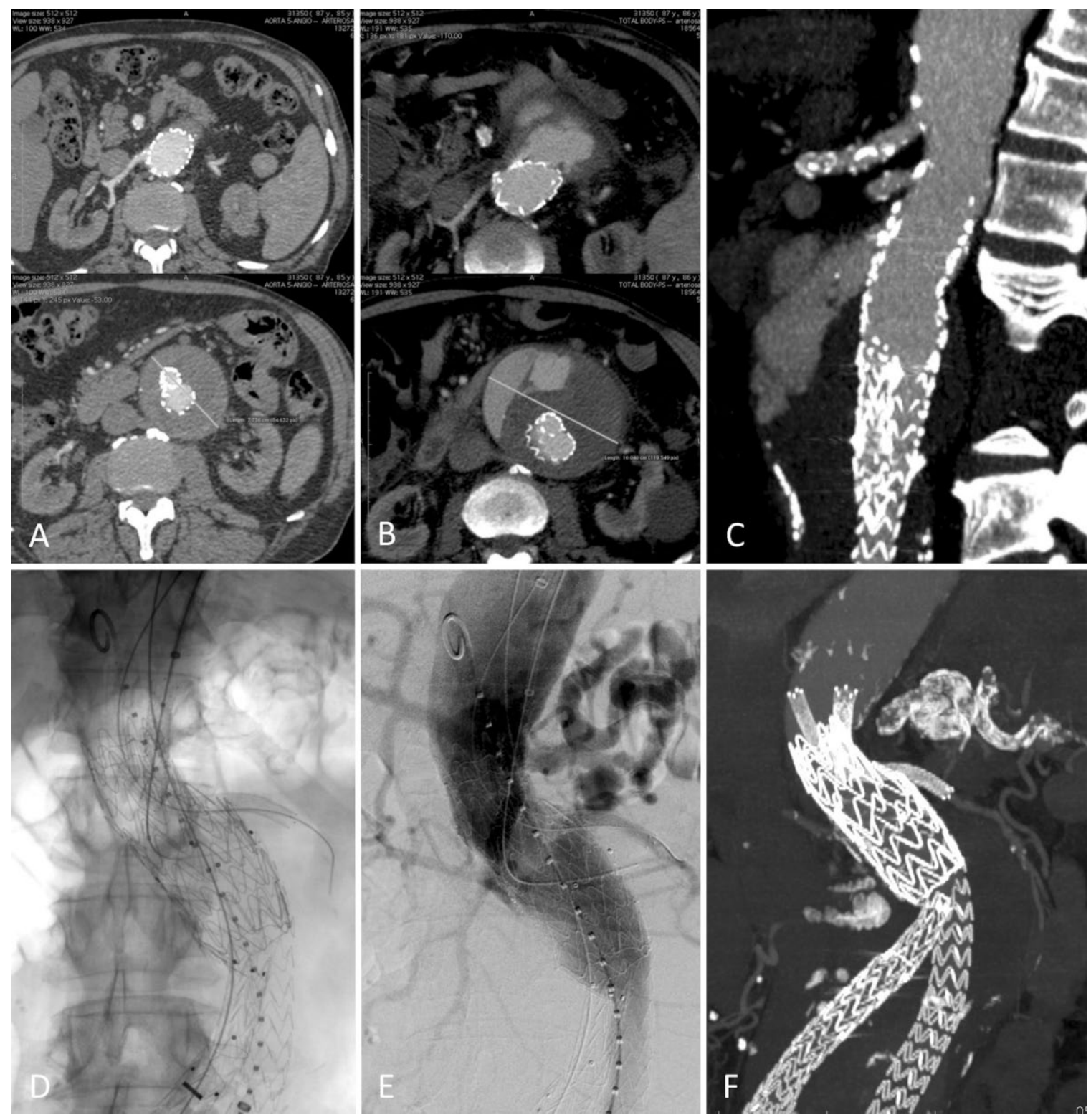

Figure I. Symptomatic patient with late type la endoleak related to partial migration of an Endurant stent-graft. (A) Computed tomography (CT) at I-year follow-up after the original endovascular aneurysm repair (EVAR). (B, C) At 4 years, CT imaging showed a type la endoleak with sac expansion. (D, E) After chimney EVAR using a C-TAG stent-graft and 2 different types of chimney grafts (Viabahn for the left renal artery and Atrium Advanta for the superior mesenteric artery, both relined with bare metal stents). (F) Postoperative CT reconstruction.

No statistically significant difference was noted in terms of freedom from type Ia endoleak at follow-up between chEVAR performed using an Endurant device and other devices $[1 / 20(5 \%)$ vs $2 / 19(11 \%), \mathrm{p}=0.6]$ or reintervention $[1 / 20(5 \%)$ vs $0 / 19(0 \%)]$.

The pre/post maximum sac diameters were $71.5 \pm 29.0$ $\mathrm{mm}$ and $69.9 \pm 27.5 \mathrm{~mm}$, respectively, for a $1.6-\mathrm{mm}$ postoperative sac shrinkage $(\mathrm{p}=0.486)$. The newly created sealing zone increased by $16.5 \mathrm{~mm}$. The pre/post infrarenal neck lengths were $3.9 \pm 4.0 \mathrm{~mm}$ and $20.4 \pm 4.2 \mathrm{~mm}$, respectively.

\section{Discussion}

The incidence of type Ia endoleak has been reduced during the last few years thanks to improvements in stent-graft designs and better patient selection; nonetheless, the rate is 
A

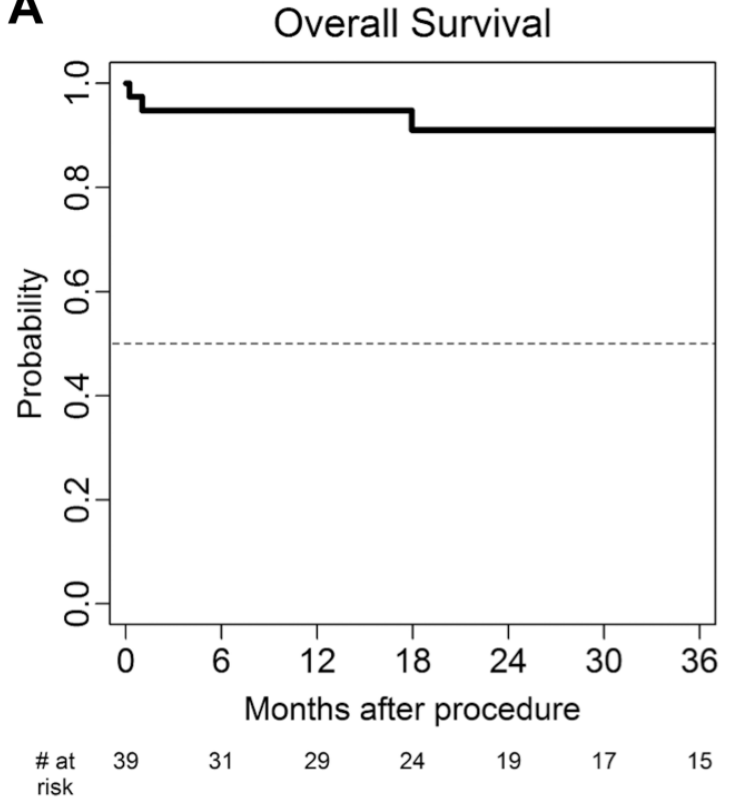

B Freedom from Occlusion

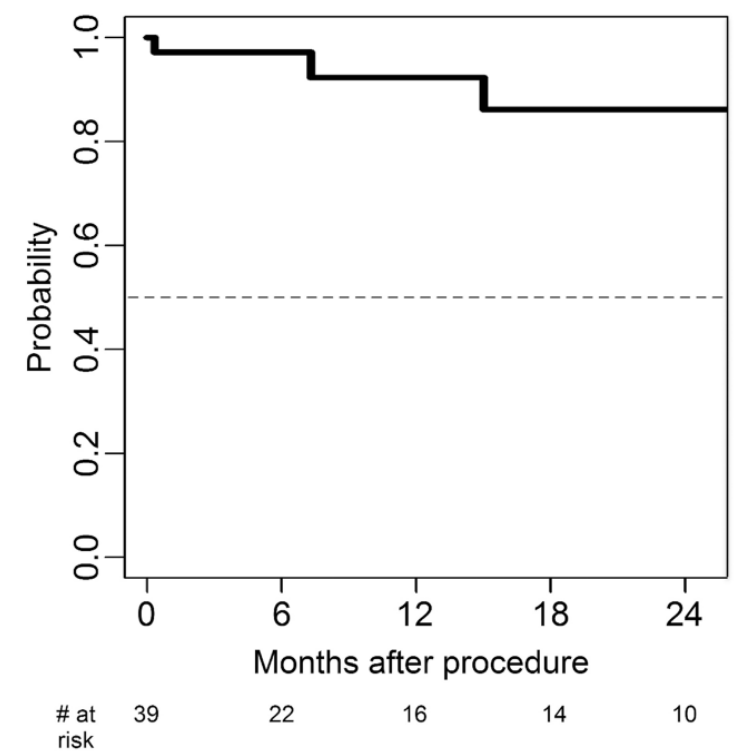

Figure 2. Kaplan-Meier curves of (A) chimney graft primary patency in 39 patients and (B) overall survival.

still not negligible, ranging from $3.5 \%$ to $15.5 \%$ in the latest series. ${ }^{1,2}$ While intraoperative type Ia endoleaks can resolve spontaneously in some cases, justifying a watchful waiting strategy, late type Ia endoleaks need to be treated because they are strictly associated with sac growth, and ultimately, to aneurysm rupture. ${ }^{14-16}$ At the moment there is no consensus about the optimal treatment.

Open conversion represents a valid and effective option, but it carries a high risk of perioperative mortality, which increases dramatically in the case of rupture. ${ }^{17-19}$ The possibility of performing a partial conversion or entirely sparing the endograft has been shown to reduce the risk of the open procedure, ${ }^{20}$ but endovascular treatment, when feasible, certainly represents a better option for this high-risk population. ${ }^{21-23}$

Cuffs are a reasonable approach to treat distal migration with a residual infrarenal neck, but different strategies are necessary when the graft is still in place. Embolization with Onyx and/or coils has been described as an effective option in selected cases. Ameli-Renani et $\mathrm{al}^{24}$ recently reported the results of 27 type Ia endoleak embolization procedures in 25 patients: despite an early technical success of $100 \%$, the recurrence rate in this series was as high as $32 \%$. The authors noted some anatomic patterns that increased the risk for late recurrence, in particular, wide endoleak cavities with a large entrance in the aneurysm sac. Moreover, in this situation there is always a certain risk of Onyx reflux. ${ }^{25}$

Endoanchors are designed to enhance endograft proximal fixation and sealing. The ANCHOR registry compared the prophylactic use of the anchors with their use in the treatment of proximal aortic neck complications and found better technical success (absence of a type Ia endoleak at completion angiography) for the former $(89.7 \%$ vs $80.5 \%$, respectively). ${ }^{11}$

Fenestrated grafts are a valid alternative to correct endoleaks alone or in associated with proximal neck evolution. However, there are technical problems related to the presence of the previously implanted stent-grafts (kinking/stenosis/tortuosity of the leg, suprarenal stent in front of the renal arteries, difficult visualization of the fenestration markers) and to the large diameter of the custom devices. The technical success of these procedures is between $58.3 \%$ and $92.3 \%$, and the immediate target vessel failure is $5.4 \%$ to $8 \%{ }^{26-28}$

The chimney technique seems a feasible and relatively safe option in endoleak treatment. The Münster group ${ }^{29}$ reported an experience with 18 EVAR patients with juxtarenal aneurysms and type I endoleak treated using primarily the Endurant stent-graft in conjunction with the Advanta stent-graft as the chimney. Primary CG patency was $94.4 \%$ and secondary patency was $100 \%$. The Zurich group ${ }^{30}$ reported 24 type I endoleaks, including some thoracoabdominal cases and 7 treated urgently ( 5 symptomatic, 2 with rupture). Of the 55 target vessels, 31 received chimneys and 24 sandwich/periscope configurations, with most cases treated using a combination of Excluder and Viabahn. Technical success was $96 \%$, but all target vessels were revascularized. The estimated parallel graft patency was $94 \%$. Tanious et $\mathrm{al}^{31}$ treated 19 cases with $100 \%$ technical 
success and CG patency at 17 months. Youssef et $\mathrm{al}^{32}$ successfully treated 10 post-EVAR type I endoleaks using chimneys and the Nellix endovascular aneurysm sealing system; all repairs were patent at 8 months.

The current retrospective analysis adds further support to the efficacy of this off-the-shelf strategy, even in urgent cases ( $23 \%$ of the series). The presence of a previous suprarenal fixation did not negatively affect cannulation of the target vessel or the late patency. The rate of endoleak recurrence was low ( 3 cases), and only 1 case required correction due to sac expansion.

When compared to the entire PERICLES registry ${ }^{13}$ the results here are similar in terms of chimney patency $(94.3 \%$ vs $94.1 \%$ ) and incidence of immediate type Ia endoleak $(92.3 \%$ vs $92.1 \%)$. While there was no difference in terms of performance among the aortic components, SECS showed slightly better patency compared with BECS/BMS, which contrasts with the results from the entire PERICLES group ${ }^{33}$ in which the primary patency is better for BECS. However, the discrepancy is likely due to the small number of patients in the current analysis.

In our opinion, there are several strong points in favor of chEVAR. This technique is readily dispensable, meaning that even symptomatic and emergency type Ia endoleaks can be managed with this strategy. Access complications are few as a stent-graft with the same profile as the one implanted is used in the majority of cases. Cannulation of the target vessels from above is generally easy, especially in downward-oriented renal arteries, and not much influenced by the presence of a suprarenal stent. Furthermore, in cases of endoleak recurrence, a further reintervention with proximal extension or gutter embolization is feasible. ${ }^{12,30}$ Last but not least, suprarenal aortic coverage in chEVAR is generally short so that risk of paraplegia is low.

The chimney technique still has some issues such as parallel graft combinations, use of multiple stent-grafts, and main graft oversizing. Scali et $\mathrm{al}^{33}$ reported that use of nitinol/polyester stent-grafts with BECS is associated with better survival compared with other aortic endografts, and repairs incorporating multiple chimney subtypes are associated with increased mortality risk. The data regarding main graft oversizing are improving thanks to in vitro studies that promoted $30 \%$ oversizing as the best option even for multiple parallel grafts. On the other hand, there is a custom method ("OverSIRIX") in which the operators choose main graft oversizing directly on the CT scan during planning. ${ }^{34,35}$

\section{Conclusion}

Our experience demonstrates that the chimney technique is a safe and viable option to treat type Ia endoleak, with good midterm results, both for primary target vessel patency and resolution of endoleak. Results are independent of the used abdominal stent-grafts and chimney grafts combinations.
The technique is certainly the best choice for urgent and/or emergency cases.

\section{Authors' Note}

This study was presented as an abstract at the SVS Annual Vascular Meeting (May 31, 2017; San Diego, CA USA).

\section{Declaration of Conflicting Interests}

The author(s) declared no potential conflicts of interest with respect to the research, authorship, and/or publication of this article.

\section{Funding}

The author(s) received no financial support for the research, authorship, and/or publication of this article.

\section{ORCID iD}

Stefano Fazzini (iD https://orcid.org/0000-0002-4028-4947

\section{References}

1. Millen AM, Osman K, Antoniou GA, et al. United Kingdom outcomes of persistent intraoperative type Ia endoleak after standard endovascular aneurysm repair. J Vasc Surg. 2015;61:1185-1191.

2. Tadros RO, Faries PL, Ellozy SH, et al. The impact of stent graft evolution on the results of endovascular abdominal aortic aneurysm repair. J Vasc Surg. 2014;59:1518-1527.

3. AbuRahma AF, Campbell JE, Mousa AY, et al. Clinical outcomes for hostile versus favorable aortic neck anatomy in endovascular aortic aneurysm repair using modular devices. J Vasc Surg. 2011;54:13-21.

4. Hobo R, Kievit J, Leurs LJ, et al. Influence of severe infrarenal aortic neck angulation on complications at the proximal neck following endovascular AAA repair: a EUROSTAR study. J Endovasc Ther. 2007;14:1-11.

5. Waasdorp EJ, Vries JP, Hobo R, et al. Aneurysm diameter and proximal aortic neck diameter influence clinical outcome of endovascular abdominal aortic repair: a 4-year EUROSTAR experience. Ann Vasc Surg. 2005;19:755-761.

6. Sternbergh WC, Carter G, York JW, et al. Aortic neck angulation predicts adverse outcome with endovascular abdominal aortic aneurysm repair. J Vasc Surg. 2002;35:482-486.

7. Chaar CI, Eid R, Park T, et al. Delayed open conversions after endovascular abdominal aortic aneurysm repair. J Vasc Surg. 2012;55:1562-1569.

8. Turney EJ, Steenberge SP, Lyden SP, et al. Late graft explants in endovascular aneurysm repair. J Vasc Surg. 2014;59:886-893.

9. Kansal V, Nagpal S, Jetty P. Late open surgical conversion after endovascular abdominal aortic aneurysm repair. Eur $J$ Vasc Endovasc Surg. 2018;55:163-169.

10. Abdulrasak M, Resch T, Sonesson B, et al. The long-term durability of intra-operatively placed Palmaz stents for the treatment of type Ia endoleaks after EVAR of abdominal aortic aneurysm. Eur J Vasc Endovasc Surg. 2017;53:69-76.

11. Jordan WD, Mehta M, Varnagy D, et al. Results of the ANCHOR prospective, multicenter registry of EndoAnchors 
for type Ia endoleaks and endograft migration in patients with challenging anatomy. J Vasc Surg. 2014;60:885-892.e2.

12. Marcelin C, Le Bras Y, Petitpierre F, et al. Embolization for persistent type Ia endoleaks after chimney endovascular aneurysm repair with Onyx. Diagn Interv Imaging. 2017;98:849-855.

13. Donas KP, Lee JT, Lachat M, et al. Collected world experience about the performance of the snorkel/chimney endovascular technique in the treatment of complex aortic pathologies: the PERICLES registry. Ann Surg. 2015;262:546-553.

14. Gonçalves FB, Verhagen HJ, Vasanthananthan K, et al. Spontaneous delayed sealing in selected patients with a primary type-Ia endoleak after endovascular aneurysm repair. Eur J Vasc Endovasc Surg. 2014;48:53-59.

15. Schlösser FJ, Gusberg RJ, Dardik A, et al. Aneurysm rupture after EVAR: can the ultimate failure be predicted? Eur J Vasc Endovasc Surg. 2008;37:15-22.

16. Mangialardi N, Orrico M, Ronchey S, et al. Towards an entirely endovascular aortic world: an update of techniques and outcomes for endovascular and open treatment of type I, II, and III endoleaks. J Cardiovasc Surg (Torino). 2016;57:698-711.

17. Moulakakis KG, Dalainas I, Mylonas S, et al. Conversion to open repair after endografting for abdominal aortic aneurysm: a review of causes, incidence, results, and surgical techniques of reconstruction. J Endovasc Ther. 2010;17:694-702.

18. Abdallah IB, Batti SE, Abou-Rjeili M, et al. Open conversion after endovascular abdominal aneurysm repair: an 8 year single centre experience. Eur J Vasc Endovasc Surg. 2017;53:831-836.

19. Scali ST, Beck AW, Chang CK, et al. Defining risk and identifying predictors of mortality for open conversion after endovascular aortic aneurysms repair. J Vasc Surg. 2016;63:873-881.

20. Prusa AM, Wibmer AG, Nolz R, et al. Aortouni-iliac endografting as an alternative salvage procedure to open conversion in failed endovascular aneurysm repair. J Endovasc Ther. 2014;21:154-161.

21. Mangialardi N, Ronchey S, Orrico M, et al. Surgical conversion with graft salvage as a definitive treatment for persistent type II endoleak causing sac enlargement. J Vasc Surg. 2015;62:1437-1441.

22. Klonaris C, Lioudaki S, Katsargyris A, et al. Late open conversion after failed endovascular aortic aneurysm repair. $J$ Vasc Surg. 2014;59:291-297.

23. Marone EM, Mascia D, Coppi G, et al. Delayed open conversion after endovascular abdominal aortic aneurysm: device specific surgical approach. Eur J Vasc Endovasc Surg. 2013;45:457-464.

24. Ameli-Renani S, Pavlidis V, Morgan RA. Early and midterm outcomes after transcatheter embolization of type I endoleaks in 25 patients. J Vasc Surg. 2017;65:346-355.

25. Graif A, Vance AZ, Garcia MJ, et al. Transcatheter embolization of type I endoleaks associated with endovascular abdominal aortic aneurysm repair using ethylene vinyl alcohol copolymer. Vasc Endovascular Surg. 2017;51:28-32.

26. Katsargyris A, Yazar O, Oikonomou K, et al. Fenestrated stent-grafts for salvage of prior endovascular abdominal aortic aneurysm repair. Eur J Vasc Endovasc Surg. 2013;46:49-56.

27. Martin Z, Greenberg RK, Mastracci TM, et al. Late rescue of proximal endograft failure using fenestrated and branched devices. J Vasc Surg. 2014;59:1479-1487.

28. Falkensammer J, Taher F, Uhlmann M, et al. Rescue of failed endovascular aortic aneurysm repair using the fenestrated Anaconda device. J Vasc Surg. 2017;66:1334-1339.

29. Donas KP, Telve D, Torsello G, et al. Use of parallel grafts to save failed prior endovascular aortic aneurysm repair and type Ia endoleaks. J Vasc Surg. 2015;62:578-584.

30. Montelione N, Pecoraro F, Puippe G, et al. A 12-year experience with chimney and periscope grafts for treatment of type I endoleaks. $J$ Endovasc Ther. 2015;22:568-574.

31. Tanious A, Wooster M, Jung A, et al. Endovascular management of proximal fixation loss using parallel stent grafting techniques to preserve visceral flow. Ann Vasc Surg. 2017;42:169-175.

32. Youssef M, Zerwes S, Jakob R, et al. Endovascular aneurysm sealing (EVAS) and chimney EVAS in the treatment of failed endovascular aneurysm repairs. $J$ Endovasc Ther. 2017;24:115-120.

33. Scali ST, Beck AW, Torsello G, et al. Identification of optimal device combinations for the chimney endovascular aneurysm repair technique within the PERICLES registry [published online January 27, 2018]. J Vasc Surg. doi:10.1016/j.jvs.2017.10.080.

34. Mestres G, Yugueros X, Apodaka A, et al. The best in vitro conditions for two and three parallel stenting during endovascular aneurysm repair. J Vasc Surg. 2017;66:1227-1235.

35. Fazzini S, Ronchey S, Orrico M, et al. "Over-SIRIX": A new method for sizing aortic endografts in combination with the chimney grafts: early experience with aortic arch disease. Ann Vasc Surg. 2018;46:285-298. 